\title{
A Method to Determine Energy and Power of a Fracture Process in Polycrystals by Means of HVTEM
}

\author{
Pedro Alejandro Tamayo-Meza, ${ }^{1}$ Viacheslav Alexandrovich Yermishkin, ${ }^{2}$ \\ Juan Manuel Sandoval-Pineda, ${ }^{1}$ Luis Armando Flores-Herrera, ${ }^{1}$ \\ and Ricardo Gustavo Rodríguez-Cañizo ${ }^{1}$ \\ ${ }^{1}$ Instituto Politécnico Nacional, Escuela Superior de Ingeniería Mecánica y Eléctrica, Sección de Estudios de Posgrado e Investigación, \\ Laboratorio de Fuerza Atómica Avenida de las Granjas No. 682, 02250 Azcapotzalco, DF, Mexico \\ ${ }^{2}$ Institute of Metallurgy BAIKOV, Russian Academy of Science, Lenninsky Prozpekt No. 49, Moscow, Russia \\ Correspondence should be addressed to Pedro Alejandro Tamayo-Meza; ptamayom@ipn.mx
}

Received 1 October 2013; Revised 23 December 2013; Accepted 30 December 2013; Published 17 February 2014

Academic Editor: Rui Zhang

Copyright ( 2014 Pedro Alejandro Tamayo-Meza et al. This is an open access article distributed under the Creative Commons Attribution License, which permits unrestricted use, distribution, and reproduction in any medium, provided the original work is properly cited.

\begin{abstract}
Main crack propagation process in polycrystalline materials is very complex due to intercrystalline sliding mechanism effects. Multiple factors make it considerably difficult to analyze the state of stresses in the crack tip within the theoretical scope of linear fracture mechanics (LFM). As a basis for the suggested Bilby-Cottrell-Swinden model, we propose a method to determine the state of stresses in the tip of a propagating crack in microspecimens subjected to tension and measured inside a high voltage transmission electron microscope (HVTEM). Based on the electron microscopy observations of a main crack and the formation of microcracks in the plastic zone, the $J$-integral was determined.
\end{abstract}

\section{Introduction}

The complex nature of crack propagation in polycrystalline materials is explained by the intergranular sliding mechanism effect. The presence of the three modes of fracture, formation of deep microcracks of extended distances along the grain boundaries, crack ramification in grain intersections, and grain fragmentation in the plastic zone of the main crack, caused by the undeniable influence of the interface structure on the kinetics of grain sliding during the crack incubation process, all these characteristics of crack development in polycrystalline materials create a very complex scenario for a detailed analysis of the state of stresses. In this case the stresses are being developed in the close area located on the tip of the crack. The principles of linear fracture mechanics (LFM) are not enough for the description of this phenomena. LFM considers that the development of the plastic zone on the tip of the crack is only possible for cases when the development of the influenced zone occurs at a reduced scale, that is, when the extension of the plastic zone is insignificant compared to the length of the crack. Generally, this requirement is not reached during the development of a fracture because of the intergranular sliding mechanism.

Having such a diversity of the geometric characteristics in the crack initiation observed in polycrystalline materials, it is possible to select crack configurations for which the Bilby-Cottrell-Swinden (BCS) model is applicable $[1,2]$. In the present work, this model was used to analyze fractures in microspecimens of VT-51 titanium subjected to a tensile stress inside a HVTEM-JEOL 1000 and to determine the state of stresses located in the tip of the propagating crack under three different modes of fracture.

\section{Materials and Methods}

In this work, we used the model proposed by Bilby, Cottrell, and Swinden (the BCS model) which allows the analysis of the fracture process in VT-51 titanium alloy microspecimens. During tests, we have measured length and the components of the displacement vectors $u, v$, and $w$ of the crack tip. 


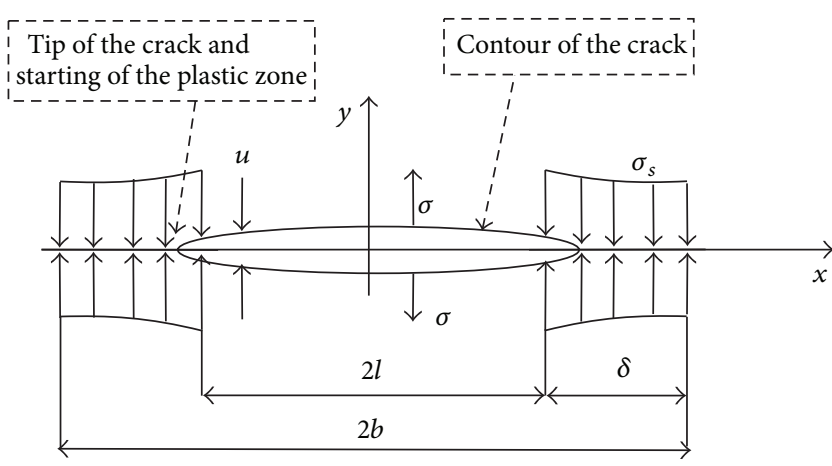

FIGURE 1: Fundamental parameters for determining the propagation of a crack according to the BCS model $[1,4]$.

The BCS model allows the study of cracks developed under different conditions, that is, the conditions of transversal slip (Mode II) or longitudinal slip (Mode III). Nevertheless, the results obtained by Dugdale [3] in the analysis of the fracture development under normal release, both ideologically and formally, are very close to the fundamental generalizations emerged from the BCS model. For this reason, it is possible to analyze the growth of a crack by using some combination of fracture modes. In this sense, we use the general case of the BCS model superimposing the three crack modes.

\section{Theoretical Analysis of the Problem}

In order to determine the $\delta_{u, v, w}$ components that describe the propagation displacements during the opening of a crack, the following equations were obtained based on the BCS model $[1,3-5]$ :

$$
\begin{gathered}
\delta_{u}=\frac{8\left(1-\nu^{2}\right) \sigma_{s} l}{\pi E} \ln \frac{b}{l}, \quad \text { for crack Mode I, } \\
\delta_{\nu}=\frac{8 \sigma_{s} l}{\pi E} \ln \frac{b}{l}, \quad \text { for crack Mode II, } \\
\delta_{w}=\frac{8(1+\nu) \sigma_{s} l}{\pi E} \ln \frac{b}{l}, \quad \text { for crack Mode III, }
\end{gathered}
$$

where $\sigma_{s}$ is the material's yield strength, $l$ is the average crack length, $b$ is the average length of the crack with the plastic zone, $v$ is the Poisson's ratio, and $E$ is Young's modulus. The scheme explaining the physical meaning of the fundamental parameters involved in (1) is presented in Figure 1 for an elliptic-shape crack.

The geometric characteristics of $b$ and $l$ are related to the applied stress by means of Dugdale's relation $[2,4]$ :

$$
\frac{l}{b}=\cos \frac{\pi \sigma}{2 \sigma_{s}}
$$

According to [4], the stress intensity factor can be calculated with

$$
K_{i}=\sqrt{E^{\prime} \cdot \sigma_{s} \delta_{i}}
$$

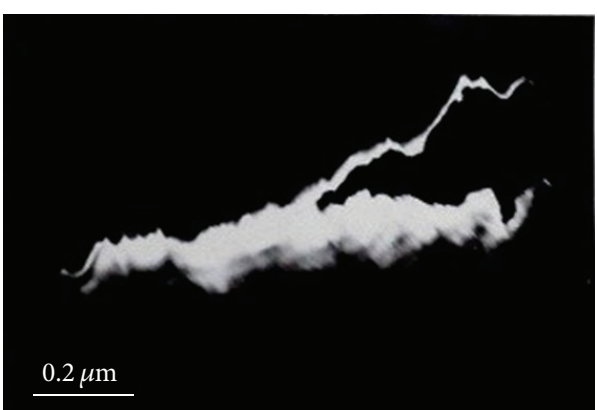

FIGURE 2: Development of a crack in VT51 titanium alloy in hardened state during mechanical and thermomechanical treatment.

where $E^{\prime}=E$ for the case of plane strain, $E^{\prime}=E /\left(1-\nu^{2}\right)$ for the case of plane deformation, and $K_{i}$ is the stress intensity factor corresponding to a particular crack mode. The $K_{i}$ values were calculated by substituting in (3), the corresponding values of $\delta_{i}$. These values were obtained from micrographs coming from a set of cracks that were experimentally generated inside the HTVEM. From them and by using (4), we determine the $J$-integral.

The $J$-integral is the energy released under a particular condition; a plastic zone is created just from the tip of the crack [4]:

$$
\begin{gathered}
J=\frac{1-v^{2}}{E}\left(K_{\mathrm{I}}^{2}+K_{\mathrm{II}}^{2}\right)+\frac{1+v}{E} K_{\mathrm{III}}^{2}, \quad \text { for plane deformation, } \\
J=\frac{K_{\mathrm{I}}^{2}+K_{\mathrm{II}}^{2}}{E}+\frac{1+v}{E} K_{\mathrm{III}}^{2}, \quad \text { for plane stress, }
\end{gathered}
$$

where $K_{\mathrm{I}}, K_{\mathrm{II}}$, and $K_{\mathrm{III}}$ are the stress intensity factors for crack Modes I, II, and III, respectively. The values of Young's modulus and Poisson's ratio in (3) and (4) were taken from [6], but $\sigma_{s}$ and $b$ were calculated from the measurements of $\delta_{u}$ and $\delta_{\gamma}$. Equation (1) was used to verify the values of $\sigma_{s}$ and $b$. Figure 2 shows the development of an intergranular crack on a VT-51 alloy microspecimen in a hardened state. The crack compliance was evaluated according to the theory of fracture mechanics. The results obtained from experiments on VT-51 Titanium Alloy subjected to a tension load were used. The experimental work was carried out in situ; the tension load was applied inside the HTVEM. The microspecimens were deformed until the moment where the microcracks started to appear. During the first stage of the process the development of the crack was carried out as expected (Mode I). Next, when the load was increased, the direction of the crack followed certain angle due to transverse shearing (Mode II). The intergranular sliding process enhances the propagation of the crack, a combination of lengthwise and transversal propagation of the crack is presented and creates instability, and finally the three modes of fracture appear and this combination leads to the final destruction of the specimen.

The boundary is clearly observed in the tip of the crack; it is the place where the sliding occurs. A close view of the main crack and the development of the microcrack are shown in Figure 3. 


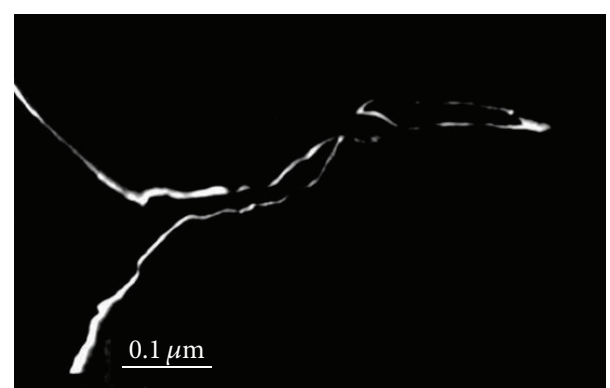

FIGURE 3: Development of a microcrack on the tip of the embedded main crack in the VT-51 titanium alloy.

The characteristics of the $J$ energy and the $K_{i}$ stress for a VT-51 alloy are shown in Table 1 . They were obtained in situ from the HVTEM during the development of the crack.

In this table, number 1 corresponds to the crack shown in Figure 4(a), and number 2 corresponds to the crack shown in Figure 4(b).

\section{Discussion}

The data in Table 1 reveal that, after the thermomechanical treatment, the energy of the crack process is increased by one order. In particular, as the energy increases, the resistance to the intergranular sliding $K_{\mathrm{III}}$ also increases, and the length of the plastic zone in the tip of the crack is reduced by one order. Figure 5 demonstrates the variation of the crack propagation velocity and the value of the $J$-integral starting from the moment of the increment of the load and until the cycle load is completed. In this figure we observe the first 7 seconds after the microspecimen is released, the crack activates its development, and the energy of the process is slowly increased. The subsequent increment of the crack process is followed by a sensible decrement of the crack propagation velocity which is kept at a constant level even after the moment when the energy of the crack development is decreased. At this stage, the midsection of the crack is significantly increased. At the next moment, which is the cycle of growth acceleration of the crack, a small kink is formed in the tip. This sequence of events in the development of the crack is observed under the stress relaxation, as well as for a constant load condition.

The pattern obtained from the test influences only the kinetics of these events. It should be pointed out that the data obtained for $\delta$ according to (1) are very close to the ones obtained from the experiment.

In general, the $J$-integral is determined by the released potential of the elastic energy intensity, according to the following expression [4]:

$$
J=-\frac{\partial \Pi}{\partial l}
$$

This equation is the basis for the development of the experimental methods intended for determining the $J$-integral. In particular, Mughrabi [7] proposed a simple method to determine the $J$-integral using the results of bending tests on plane specimens. He obtained the following expression:

$$
J=\frac{2 \int_{0}^{\delta_{c}} P d \delta_{c}}{B b},
$$

where $\int_{0}^{\delta_{c}} P d \delta_{c}$ is the surface under the load versus the displacement curve, $B$ is the specimen thickness, and $b$ is the length of the specimen before the test. Nevertheless, considering the fact that the loading device, which carries out the deformation process inside an HVTEM, operates according to the uniaxial stress scheme, it is more convenient to use the equation proposed by Mughrabi [7] to determine the $J$-integral for the in situ experiments. This equation in a modified form can be applied as follows:

$$
\begin{gathered}
J=\frac{2 \int_{0}^{\delta_{c}} P d \delta_{c}}{B b}\left[\frac{1+\alpha}{1+\alpha^{2}}\right], \\
\text { where } \alpha=\sqrt{\left(\frac{2 l_{0}}{b}\right)^{2}+2\left(\frac{l_{0}}{b}\right)+2}-\frac{2 l_{0}}{b+1},
\end{gathered}
$$

where $l_{0}$ is the initial length of the crack. Taking all above into account, if the initial crack length is considerable compared to $b$, it is possible to observe that (7) approximates to (6). At the same time, application of (7) for determining the $J$-integral and using the experimental data obtained in the HVTEM does not eliminate all of the characteristic problems for the following reasons.

(a) It is very difficult to determine the integral $\int_{0}^{\delta_{c}} P d \delta_{c}$ if the grips for a tensile test on microspecimens do not have an attachment for plotting a " $P-\delta_{c}$ " curve.

(b) Microcracks initiated in specimens during a tensile test inside an HVTEM are developed because the three crack modes are combined, but (7) considers that the test on a specimen is carried out only by a normal breakaway.

Due to the above, an approach to avoid these difficulties is necessary.

\section{Proposal of Solution}

We developed an approach to define the $J$-integral from the data obtained experimentally during tensile tests applied to microspecimens in a standard grip system inside the column of an HVTEM. Our approach considers the contribution of the all three crack modes in a single magnitude of the $J$-integral: $J=G_{\mathrm{I}}+G_{\mathrm{II}}+G_{\mathrm{III}}$; according to [8], the velocity of the deformation energy release during the crack development process can be expressed as in

$$
G=P \cdot \frac{d u}{d t}-\frac{d V}{d l}
$$

where $d l$ is the increment of the crack, $u$ is the sliding from the point where the $P$ force is applied, and $V=P u / 2$ is the elastic energy released during the crack development process. 
TABLE 1: Characteristics of energy, power and geometry during the development of a crack in a VT-51 titanium alloy.

\begin{tabular}{lccccccccc}
\hline Crack no. & $\delta_{u}, \mu \mathrm{m}$ & $\delta_{v}, \mu \mathrm{m}$ & $\delta_{w}, \mu \mathrm{m}$ & $b, \mu \mathrm{m}$ & $l, \mu \mathrm{m}$ & $K_{\mathrm{I}}, \mathrm{MPa} \sqrt{\mathrm{M}}$ & $K_{\mathrm{II}}, \mathrm{MPa} \sqrt{\mathrm{M}}$ & $K_{\mathrm{III}}, \mathrm{MPa} \sqrt{\mathrm{M}}$ & $J, \mathrm{MJoul} / \mathrm{M}^{2}$ \\
\hline 1 & 0,098 & 0,069 & 0,141 & 5,76 & 3,51 & 1,71 & 1,43 & 2,05 & 1,27 \\
2 & 0,052 & 0,052 & 0,730 & 14,68 & 12,94 & 1,78 & 1,78 & 6,71 & 14,73 \\
\hline
\end{tabular}

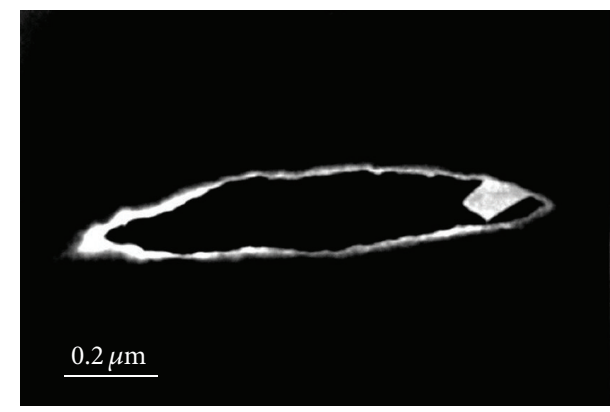

(a)

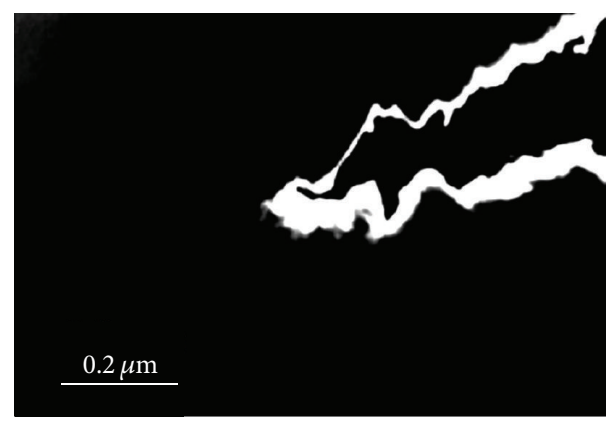

(b)

FIGURE 4: Development of an intergranular crack in a VT-51 titanium alloy, (a) in tempered state and (b) in hardened state.

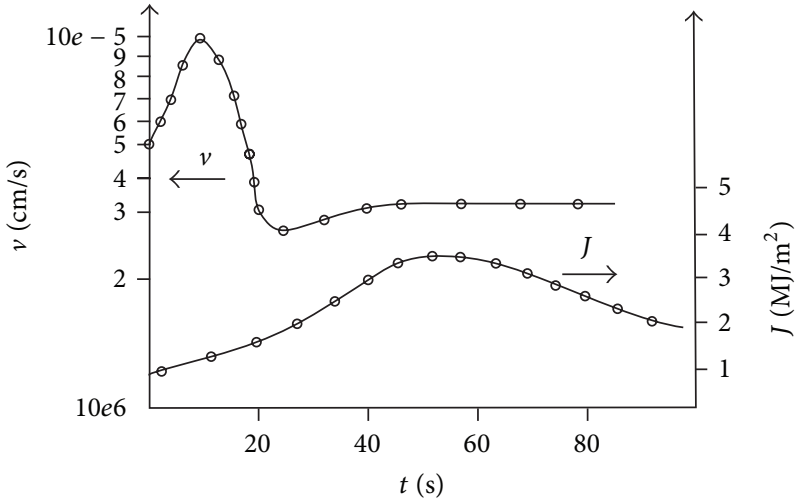

FIGURE 5: Kinetics of the crack propagation and the crack energy in a VT-51 alloy.

Considering that $V$ may be expressed via the load and according to the fact that $V=P^{2} c / 2$, then the derivative $d u / d l$ in (8) is determined as in

$$
\frac{d u}{d l}=\frac{\alpha(c P)}{d l}=P \frac{d c}{d l}+c \cdot \frac{d P}{d l} .
$$

Equation (8) can be expressed as in

$$
G=\frac{P^{2}}{2}\left(\frac{d c}{d l}\right)
$$

Considering the thickness $t$ of the specimen, (9) can be rewritten as

$$
G=\frac{P^{2}}{2 t}\left(\frac{d c}{d l}\right)
$$

If a load applied to a microspecimen produces a crack development by means of intergranular sliding along the boundaries, the direction of the load is described considering $\cos \alpha_{1}$; $\cos \alpha_{2} ; \cos \alpha_{3}$, as shown in Figure 6, and with a displacement in the direction of the loads $P \cdot \cos \alpha_{1} ; P \cdot \cos \alpha_{2} ; P \cdot \cos \alpha_{3}$, which is equal to

$$
\begin{gathered}
u=\Delta L \cos \alpha_{1} \cdot c, \quad V=\Delta L \cos \alpha_{2} \cdot c, \\
W=\Delta L \cos \alpha_{3} \cdot c,
\end{gathered}
$$

respectively, and then the value of the $J$-integral can be expressed as in

$$
J=\sum_{i=1}^{n=3} \frac{P^{2}}{2 t} \cdot \cos ^{2} \alpha_{i} \frac{d c}{d l}
$$

where $c$ is the crack compliance deformation caused by the loading device which is acting on the specimen.

The direction of the cosines can be determined if the stress direction and the direction of the displacement of the boundaries during the process of the crack formation are known. The values of load $P$ for a given crack length are assigned from the desired calibration graph " $P-u$ " of the device by fulfillment of the hypothesis that the edges of the microspecimen remain plane during the deformation.

In cases when a microcrack is initiated in the main tip of the crack, it is possible to use the approach known as the HRR method to determine the $J$-integral which describes the development of a main crack [4, 7-10]. Considering the results of Hutchinson et al. $[4,9,10]$ and Hutchinson [11], it is possible to obtain an expression for the calculation of the stress field at the tip of the crack:

$$
\frac{\sigma_{i j}\left(r_{1} \theta\right)}{\sigma_{s}}=\left(\frac{J E}{I \alpha \cdot \sigma_{s}^{2}}\right)^{1 /(n+1)} \cdot \frac{1}{(r / l)^{1 /(n+1)}} \cdot \widetilde{\sigma}_{i j}(\theta),
$$

where $I$ is the nondimensional coefficient determined by numerical methods [9], $\alpha$ is the coefficient in the order of the function which describes the deformation curve as in

$$
\frac{\varepsilon_{n}}{\varepsilon_{s}}=\alpha\left(\frac{\sigma}{\sigma_{s}}\right)^{n},
$$




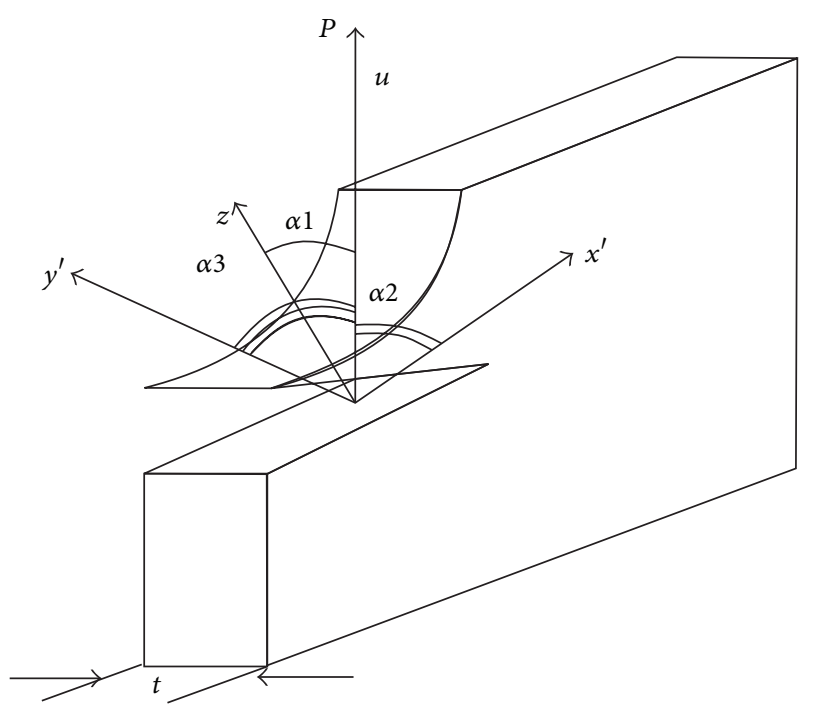

FIGURE 6: Formation of the crack due to intergranular sliding.

$n$ is the exponent in the function, $\sigma_{s}$ is the material yield stress, $r$ is the length of a radius vector which connects the crack tip with the point where the stress state is determined, and $\widetilde{\sigma}_{i j}(\theta)$ is the normal trigonometric function of the azimuthal angle of the radius vector.

The $J$-integral located along a contour of radius $r_{2}$, which surrounds the plastic zone, can be evaluated according to Mughrabi et al. [7, 9] using

$$
\begin{gathered}
J=\alpha K^{n+1} \cdot r_{2}{ }^{(n+1) \cdot(s-2)+1} \cdot I, \\
\text { where } s=\frac{2 n+1}{n+1} .
\end{gathered}
$$

The graphs of the function $\widetilde{\sigma}_{i f}(\theta)$, plotted by means of numerical methods, are known only for the crack mode under normal breakaway conditions and for longitudinal displacement $[9,10]$. Nevertheless, considering that the superposition principle is not applicable to elements in the plastic state [11], it is not possible to theoretically describe the $\widetilde{\sigma}_{i f}(\theta)$ function for a general case of fracture development and under the conditions of a combination of the three crack modes, by the simple superposition of partial solutions for each one of these modes. The proposed methodology is intended to be used for the experimental determination of the $J$-integral when a microcrack of reduced magnitudes is initiated in the front side of a main crack. First we measure the perpendicular directions of the crack tip (1) on the basis of the BCS model, and the components $\sigma_{x x}, \sigma_{y y}$, and $\tau_{x y}$ of the state of stress in the location of microcracks are determined. We define the force tensor, which describes the state of stresses of the point where the microcracks are present; in a polar coordinate system with center in the main crack and its axis parallel to the crack, we carry out the transformation:

$$
\left(\begin{array}{ll}
\sigma_{x x} & \tau_{x y} \\
\tau_{y x} & \sigma_{y y}
\end{array}\right) \Longrightarrow\left(\begin{array}{cc}
\sigma_{r r} & \tau_{r \theta} \\
\tau_{\theta r} & \sigma_{\theta \theta}
\end{array}\right)
$$

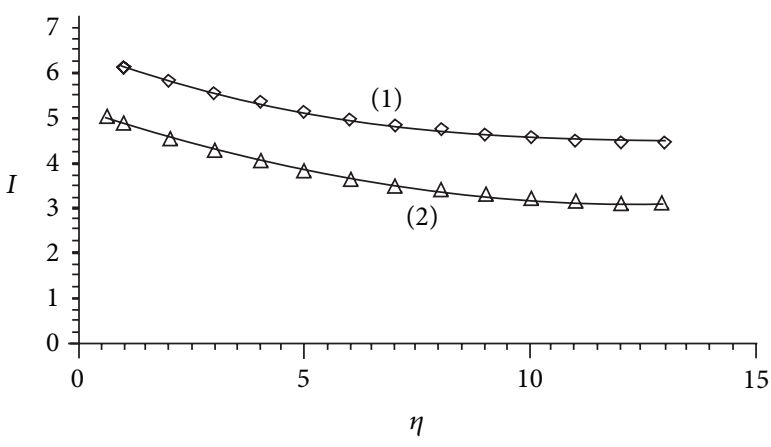

FIGURE 7: $I$ parameter as a function of the strain hardening $\eta$. (1) Plane stress and (2) plane strain [20].

With the set of $(17),[12,13]$ :

$$
\begin{gathered}
\sigma_{r r}=\frac{1}{2}\left(\sigma_{x x}+\sigma_{y y}\right)+\sqrt{\left(\frac{\sigma_{x x}-\sigma_{y y}}{2}\right)^{2}+\tau_{x y}^{2}} \cdot \cos 2 \theta, \\
\sigma_{\theta \theta}=\frac{1}{2}\left(\sigma_{x x}+\sigma_{y y}\right)-\sqrt{\frac{\left(\sigma_{x x}-\sigma_{y y}\right)^{2}}{2}+\tau_{x y}^{2}} \cdot \operatorname{sen} 2 \theta, \\
\tau_{r \theta}=\sqrt{\frac{\left(\left(\sigma_{x x}-\sigma_{y y}\right)^{2}\right)}{2}+\tau_{x y}^{2}} \cdot \operatorname{sen} 2 \theta .
\end{gathered}
$$

Using the components of the stress tensor in (14), we calculate $\sigma_{e}$ according to Von Misses, with (18), [9-11]:

$$
\sigma_{e}^{2}=\sigma_{r r}^{2}+\sigma_{\theta \theta}^{2}-\sigma_{r r} \cdot \sigma_{\theta \theta}+3 \tau_{r \theta}^{2} .
$$

Furthermore, we determine the values of the $J$-integral with the help of the generalized compliance method, (9) and (10), and after substituting $\sigma_{e}$, we calculate the values of the $\widetilde{\sigma}_{i j}(\theta)$ function.

Considering the fact that the center of a microcrack remains immovable during the evolution of the microcrack, we can use (10) and the solution of Hutchinson-RiceRosengreen for determining the $J$-integral of the main crack [14-16].

It is worth to mention that the approximated parameters of the stress curve with an exponential function of $\alpha$ and $\eta$, required to determine also the stress according to (10), can be obtained by a statistic processing of the experimental curves of the material under tension and the values of the coefficient $I$ which are determined from the graph presented in Figure 7 $[2,17-19]$.

\section{Conclusions}

As it was demonstrated by our deformation tests on polycrystalline microspecimens of VT51 titanium the micromechanism which rules fracture is the intergranular sliding. In large-grain polycrystals, fracture development at the grain boundaries as a result of intergranular sliding is accompanied by a transcrystalline break through the dislocation sliding mechanism which is a characteristic for fracture in 
monocrystals. In small-grain polycrystals, with the grain inferior diameter of $1-3 \mu \mathrm{m}$, and in polycrystals with the matrix which can be hardened by thermomechanical treatment, the effect of the dislocation sliding mechanism may be totally invoked and the whole path of the main crack is located at the grain boundaries. The final formation of a main crack took place by means of joining the microirregularities with the intergranular crack. The described fracture mechanism can be identified as the retarded sliding mechanism. Considering the fact that the fracture in polycrystals develops mostly at the grain boundaries oriented at an angle with respect to the specimen axis, the crack growth is given under the conditions of a combination of the three fracture modes: normal detachment, transversal slip, and longitudinal slip. As our observations demonstrated, the crack ramification is generally related to the main crack initiation at a triple point, that is, where the microcracks are absorbed at the location where the grains contact each other. On the basis of the proposed BCS model, we suggest a method to analyze the localized load state in the node of a developing crack and to determine the energy characteristics of its growth under the conditions of a combination of the three fracture modes using the parameters determined from the data obtained in the observations of tests fulfilled in situ inside an HVTEM column. The experimental method was verified in VT51 titanium alloy in the initial and hardened state. On the basis of the experimental method to determine the $J$-integral from the data obtained in a study of the compliance specimens with cracks, we developed a method to determine the J-integral from the analysis of the observations obtained from an HVTEM applicable to polycrystalline microspecimen under the combination of the three fracture modes.

\section{Conflict of Interests}

The authors declare that there is no conflict of interests regarding the publication of this paper.

\section{Acknowledgments}

The authors give thanks to the Instituto Politécnico Nacional, Escuela Superior de Ingeniería Mecánica y Eléctrica Unidad Azcapotzalco and the Consejo Nacional de Ciencia y Tecnología (CONACYT) in Mexico for their support during the development of this academic research work.

\section{References}

[1] J. Meyz, Theory and Problems of Continuum Mechanics, Librokom, Moscow, Russia, 3rd edition, 2010.

[2] G. G. Chell, "Bilby, Cottrell and Swinden model solutions for centre and edge cracked plates subject to arbitrary mode I loading," International Journal of Fracture, vol. 12, no. 1, pp. 135147, 1976.

[3] D. S. Dugdale, "Yielding of steel sheets containing slits," Journal of the Mechanics and Physics of Solids, vol. 8, no. 2, pp. 100-104, 1960.

[4] M. Shiratori, T. Miyoshi, and H. Matsushita, Numerical Fracture Mechanics, Jikkyo Shuppan, Tokyo, Japan, 1980.
[5] V. Z. Parton and E. M. Morozov, Elastic-Plastic Fracture Mechanics, Mir, Moscow, Russia, 1974.

[6] D. Broek, Elementary Engineering Fracture Mechanics, Nijholf, The Hague, The Netherlands, 1982.

[7] H. Mughrabi, "Observations of dislocations in the stressapplied state in copper single crystals deformed at the low temperature $\left(78^{\circ} \mathrm{K}\right)$, , in Proceedings of the Septieme Congress Internationale de Microscopie Electronique, pp. 267-268, Grenoble, France, 1970.

[8] R. W. Hertzberg, Deformation and Fracture Mechanics of Engineering Materials, John Wiley \& Sons, New York, NY, USA, 4th edition, 1996.

[9] C. W. Smith and A. S. Kobayashi, "Experimental Fracture Mechanics," in Handbook on Experimental Mechanics, chapter 20, pp. 905-968, Wiley-VCH, New York, NY, USA, 2nd edition, 1993.

[10] J. W. Hutchinson, "Singular behaviour at the end of a tensile crack in a hardening material," Journal of the Mechanics and Physics of Solids, vol. 16, no. 1, pp. 13-31, 1968.

[11] J. W. Hutchinson, "Plastic stress and strain fields at a crack tip," Journal of the Mechanics and Physics of Solids, vol. 16, no. 5, pp. 337-342, 1968.

[12] F. McClintock, Mechanical Behavior of Materials, Addison-Wesley, Boston, Mass, USA, 1966.

[13] S. P. Timoshenko and J. Gere, Mechanics of Materials, PWS Engineering, Boston, Mass, USA, 2nd edition, 1984.

[14] Y. N. Robotnov, Mechanics of a Deformed Solid Body, Nauka, Moscow, Russia, 1988.

[15] J. R. Rice, "A path independent integral and the approximate analysis of strain concentration by notches and cracks," Journal of Applied Mechanics, vol. 35, pp. 379-386, 1968.

[16] J. R. Rice and M. A. Johnson, The Role of Large Crack Tip Geometry Changes in Plane Strain Fracture, Division of Engineering, Brown University, U.S. Atomic Energy Commission, no. 38, pp. 38, 1969.

[17] B. A. Bilby, A. H. Cottrell, and A. H. Swindon, "The spread of plastic yield from a notch," Proceedings of the Royal Society A, vol. 272, no. 1350, pp. 304-314, 1963.

[18] G. Galatenko, “The duglade's type crack model for elliptic crack at multiaxial loading," in Proceedings of the 8th International Fracture Conference, pp. 51-56, Istanbul, Turkey, November 2007.

[19] D. Häussler, M. Bartsch, U. Messerschmidt, and B. Reppich, "HVTEM in situ observations of dislocation motion in the oxide dispersion strengthened superalloy MA 754," Acta Materialia, vol. 49, no. 18, pp. 3647-3657, 2001.

[20] V. F. Sujovarov, Intermitent Segregation of Fases in Alloys, Nauka, Novosibirsk, Russia, 1983 (Russian). 

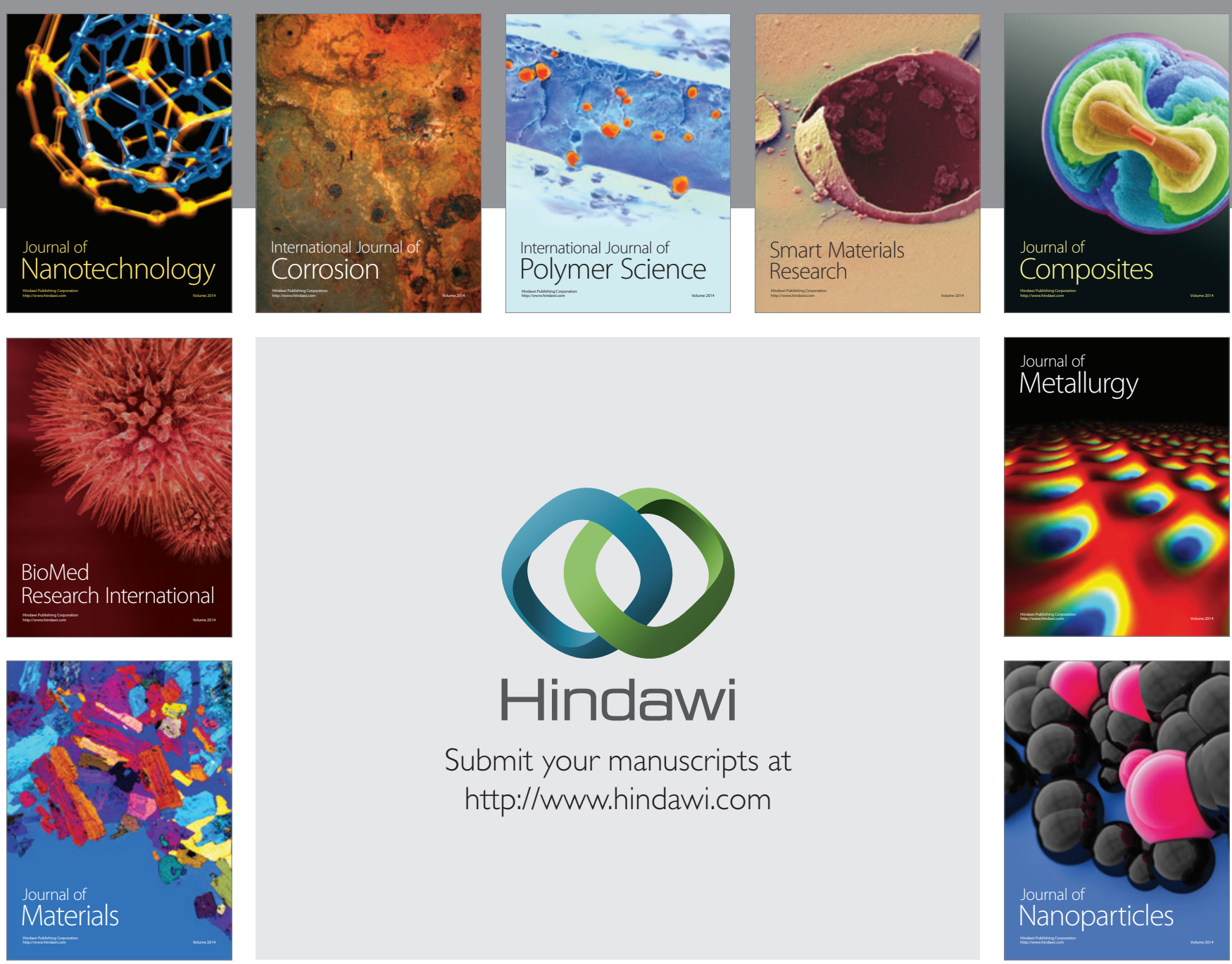

Submit your manuscripts at http://www.hindawi.com
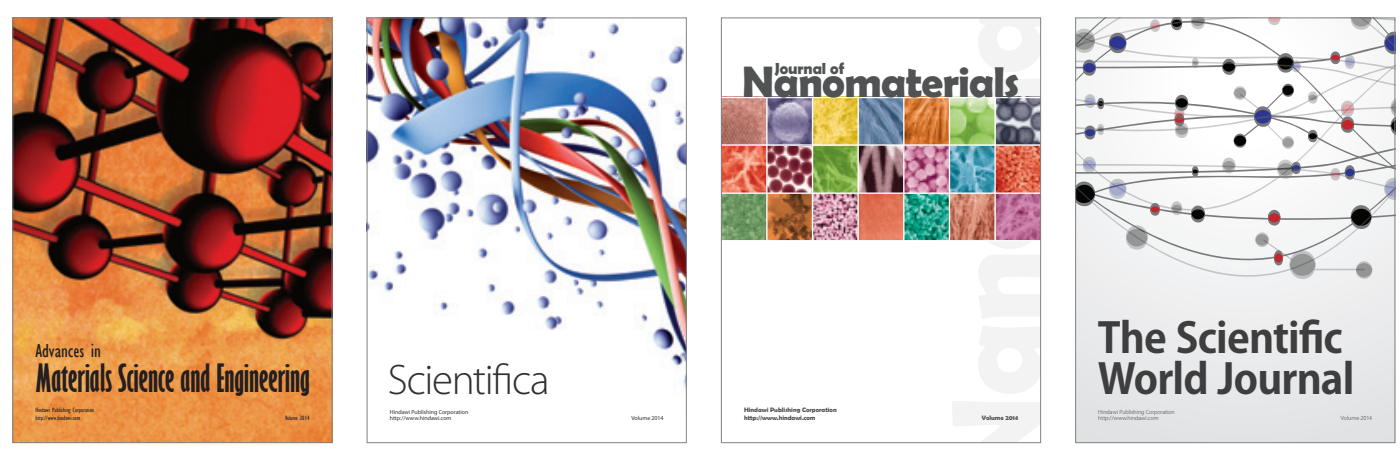

\section{The Scientific World Journal}
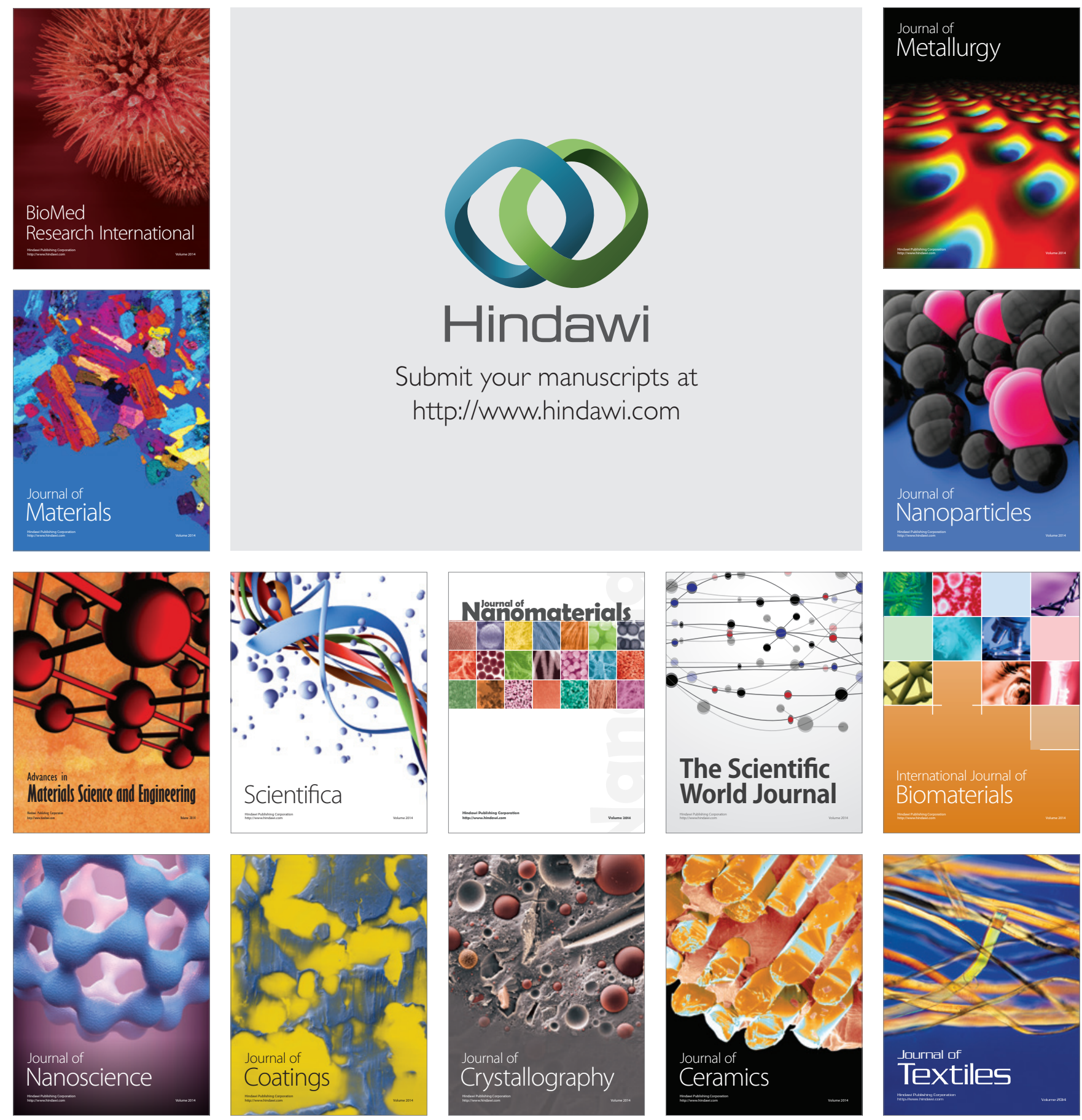